Results We were able to define 4 discrete clusters of patients based on 9 routinely collected clinical variables using data from 537 patients and 12200 encounters. Lung function outcomes were not used to define the clusters, however, there was a distinction between the different clusters, such that the cluster with the poorest outcomes also had the worst lung function. The cluster with the poorest outcomes also had the greatest risk of hospitalization and pulmonary exacerbation, which suggests that the approach correctly identifies patients with a more severe disease phenotype. The results were consistent in the GOSH clinical data.

Conclusion Four clusters of pediatric CF patients were identified with corresponding differences in clinical characteristics and outcomes. Future work will identify risk factors for transitioning to a severe disease cluster, and those factors that may improve health outcomes.

\section{INVESTIGATING TEMPORAL CHANGES IN PERCENT PREDICTED FVC AND RULM SCORE IN NON-AMBULANT SMA TYPE III CHILDREN}

${ }^{1}$ Amy Wolfe, ${ }^{2}$ Mariacristina Scoto, ${ }^{3}$ Robert Muni-Lofra, ${ }^{4}$ Evelin Milev, ${ }^{5}$ Annemarie Rohwer, ${ }^{3}$ Ruth Wake, ${ }^{3}$ Anna Mayhew, ${ }^{3}$ Chiara Marini-Bettolo, ${ }^{2}$ Francesco Muntoni. ${ }^{1}$ Great Ormond Street Hospital NHS Foundation Trust; ${ }^{2}$ UCL Great Ormond Street Institute of Child Health; ${ }^{3} J o h n$ Walton Muscular Dystrophy Research Centre, Newcastle University Translational and Clinical Research Institute, International Centre for Life, Newcastle Upon Tyne; ${ }^{4}$ Dubowitz Neuromuscular Centre, UCL Great Ormond Street Institute of Child Health; ${ }^{5}$ Great Ormond Street Hospital for Sick Children NHS Trust, London, UK

\subsection{6/archdischild-2020-gosh.84}

Spinal muscular atrophy (SMA) type III is a relatively mild form of SMA where a significant proportion lose ambulation during childhood. There is a paucity of studies investigating changes in both the respiratory and upper limb function within this population after loss of ambulation (LOA). The aim of this study is to investigate the change in the percentage of predicted forced vital capacity (FVC) and the change in the revised upper limb (RULM) score in these patients across a 24-month period after LOA. Retrospective analyses were performed on 24 non-ambulant SMA III patients on clinical data collected at two UK centres. Mean age at baseline was 10.9 years (4 to 16). The median FVC percentage predicted score at baseline was $90 \%$. We observed a significant progressive deterioration of $14.7 \%$ in FVC over the 24month period. Data on RULM was available in 16 patients with mean age at baseline of 11.5 years (6 to 16). The mean score at baseline was 30.3 . We observed a significant progressive deterioration in upper limb function over the 24 months with a mean decrease in RULM score of 3 and a range from -8 to +1 . Using a Wilcoxon signed rank test both results were significant $(\mathrm{p}<0.05)$. This study highlights that SMA type III patients demonstrate progressive deterioration in their upper limb and respiratory function even after LOA. Combining correlative data from these assessments may provide insight into clinical progression within this patient population which could help inform for clinical trials and be helpful in managing disease progression expectation for patients.
85 AUGMENTING SOFT TOYS FOR SELF-REPORTING WELLBEING

${ }^{1}$ Tu Dinh Duong, ${ }^{1}$ Cheng-Kai Yu, 'Leon Reicherts, ${ }^{2}$ Ethan Wood, ${ }^{3}$ Neil J Sebire, ${ }^{1}$ Ann Blandford, ${ }^{1} Y$ vonne Rogers. ${ }^{1}$ UCL Interaction Centre - University College London; ${ }^{2}$ UCL Department of Computer Sciences; ${ }^{3}$ GOSH DRIVE

10.1136/archdischild-2020-gosh.85

It can be difficult for children to express and reflect on how they are feeling to others. As such, we hypothesise that children can use technology to express and reflect on their wellbeing. Existing research suggests that tangible interfaces offer a potential of being able to help capture well-being in hospitals for children, such as capitalising on young children's attraction to toys and play as a way for children to communicate and process their inner thoughts.

An aim was to develop a prototype to enable children to communicate and express how they are feeling with others. To ensure that prototypes were safe and appropriate, we involved The Young Persons' Advisory Group and the Play team to provide vicarious insights about a child's experiences as part of the design process. This process helped to identify user experience challenges such as when reflecting on the potential for negative experiences. From the insights gained, we developed a system that allows children to augment existing toys to make toys interactive and give children a sense of ownership. There were two key modes of interaction, firstly the soft toy acting as the input mechanism for data to be collected. Secondly, there was a visualisation display which would then render the data for children in an accessible way for them to reflect on.

To ensure that the system was safe and appropriate before children used it, we used a 'proxy' method to evaluate the system with researchers using prototypes in the wild. It was found that by having a two-pronged approach was considered valuable: (i) the tangible nature of collecting input provided a physical experience of being able to capture and reflect on well-being at the same time, and (ii) viewing visualisations from the system had a positive impact on the self-awareness of well-being.

\section{PONSETI TECHNIQUE FOR CONGENITAL TALIPES EQUINOVARUS - A PHYSIOTHERAPY LED SERVICE}

${ }^{1}$ Henry Bowyer, ${ }^{2}$ Neil Segaren, ${ }^{2}$ Mia Dunkley, ${ }^{2}$ Evette Parnell, ${ }^{2}$ Deborah Eastwood. ${ }^{1}$ GOSH; ${ }^{2}$ Great Ormond Street Hospital

\subsection{6/archdischild-2020-gosh.86}

Background Congenital Talipes Equinovarus (CTEV) deformity affects 1:1000 new-borns. Since the Ponseti technique became popular, the need for operative intervention beyond tenotomy has reduced. However, mid-long term results remain poorly reported. This prospective case series describes our patient population and outcomes since 2011 with an innovative physiotherapy-led Ponseti service.

Methods A prospective study of CTEV patients attending the Ponseti clinic. Primary outcomes included range of movement, calf circumference and functional status. Parents of children over the age of 5 were asked to complete the Oxford Ankle Foot questionnaire (OxFAQ). 